[31]

\title{
ESTUDIO DE UN FENÓMENO DE DESPRENDIMIENTO DE ROCAS (ROCK-FALL) EN LA SIERRA DE SAN JULIÁN, (ALICANTE)
}

\section{Artemio Cuenca Payá}

INTRODUCCIÓN.- El 24 de noviembre de 1984, poco después del mediodía, se produjo un desprendimiento de rocas en el antiguo frente de cantera que la Junta de Obras del Puerto abrió en el extremo SW de la Sierra de San Julián (fig. 1) afectando a una masa de 2.800 Tm de calcarenitas cuyo impacto contra el suelo produjo un pequeño seísmo registrado en el Observatorio Sismológico de Alicante, a 1,4 km de distancia, a las 13.26.55,8 (TMG) y 6,4 segundos después en el de Canalobre, a 18 kilómetros.

Por sus características, tal y como se expondrá después, puede englobarse en la categoría de los «toppling-fall» que los autores anglosajones separan de los «debris-fall» y de los «slab-fall», siendo importante esta matización ya que en castellano el término desprendimiento implica tanto la caída de piedras más o menos individualizadas (debris-fall) como el deslizamiento de un gran prisma rocoso a través de discontinuidades (slab-fall) y la caída de un prisma separado por planos fuertemente inclinados, con rotura en la base (toppling-fall),

Procesos de tipo «debris-fall» ya habían sido detectados en los escarpes de esa Sierra con ocasión de lluvias torrenciales (Ramos Hidalgo, 1983, 1984), hasta el punto de hacer necesaria la instalación de redes y muros de recogida de piedras por parte del MOPU para proteger la carretera A-190; también hay referencias verbales de antiguos canteros en el sentido de desprendimientos catastróficos durante el período de explotación de la cantera (desde finales del siglo pasado hasta 1929) con pérdidas [32] humanas y llegando al enterramiento del tren utilizado antaño para transportar las rocas al Puerto de Alicante.

Tras el abandono de la explotación, el escarpe quedó como un elemento más del paisaje circumurbano de Alicante sin que se le prestase la menor importancia hasta que hacia 1970 se construyó un campo de fútbol al pie mismo del frente, borrándose las huellas de posibles caídas de piedras ocurridas durante cuatro décadas, fenómeno este plausible si se revisan los materiales todavía existentes, aunque removidos, al pie del cantil. Como en otros tantos casos, el peligro de un desprendimiento catastrófico quedó limitado a simples comentarios informales entre geólogos y geomorfólogos bajo el supuesto de que las entidades competentes ya lo tendrían previsto, pero aquel se produjo sorprendiendo a todos excepto a los pocos que lo habían pronosticado, permitiendo así observar un fenómeno que rara vez cuenta con testigos cuando se presenta en la Naturaleza. La descripción del mismo es el objeto del presente trabajo.

LOCALIZACIÓN.- La Sierra de San Julián, también conocida como Sierra Gorda o Serra Grossa (aunque algunos lugareños rechazan este último y limitan el de San Julián al macizo NE denominando al del SW Serra del Molinet) está formada por una serie uniforme de calcarenitas bioclásticas serravallienses (Montenat, 1975) con un espesor algo superior a ciento cincuenta metros. Al NE, en la zona de la Albufereta, se apoyan sobre margas eocenas, mientras que en la ladera NW emergen unas margas burdigalienses-langhienses cuya relación con las calcarenitas no queda clara pues el contacto está oculto.

En el extremo SW, donde se abre el frente de cantera en sentido NW-SE, las capas calcareníticas se orientan N-60 -E buzando entre $15^{\circ}$ y $20^{\circ}$ al SE. Una suave discordancia se aprecia hacia la mitad del frente de forma que el tramo inferior termina con unos tres metros de areniscas y margas arenosas en capas alternantes, pasando localmente a margas grises, sobre las que es transgresivo el tramo superior de litología idéntica a la del resto del tramo inferior. Un sistema de fallas normales de salto decimétrico, con intervalos a veces inferiores al metro, se 
orientan entre $\mathrm{N}-10^{\mathrm{a}}-\mathrm{W}$ y N-20 $\mathrm{a}-\mathrm{W}$ con buzamiento desde vertical [33] hasta $70^{\circ} \mathrm{W}$. Otro sistema pero este de diaclasas, aparece a $\mathrm{N}-60^{\circ}-\mathrm{W}$ buzando entre $90^{\circ}$ y $60^{\circ} \mathrm{SW}$.

DESARROLLO DEL PROCESO.- La inestabilidad potencial del frente de cantera era manifiesta dada la conjunción de los planos de discontinuidad con el frente, que aislaba prismas rocosos de base triangular isósceles como la presentada en la figura 2. A esto se hace necesario sumar la existencia de una grieta en coronación abierta según el sistema de fallas, con anchura variable entre 10 y $30 \mathrm{~cm}$, y cuyo despegue venía facilitado por desplazamientos relativos horizontales aprovechando el sistema de diaclasas.

Ese era el estado hasta los días 9 a 11 de noviembre durante los cuales llovieron sobre Alicante 60,1 mm repartidos con mediana regularidad, 19,0 mm el día 9, 27,5 el 10 y 13,6 el 11, lo que permitió una infiltración importante en las grietas confirmada por el hecho de que en los días siguientes aparecieron rezumes de agua al nivel de la discordancia. Comenzaron entonces también a detectarse esporádicas caídas de piedras pequeñas, de tamaño no superior a $10-15 \mathrm{~cm}$, continuando éstos hasta que el día 22, hacia las 10 (TMG), cayeron aproximadamente dos metros cúbicos de rocas fragmentadas desde la arista NW del futuro plano de rotura, fenómeno que se repitió el día 24, minutos antes de las 11 y con un volumen doble. Hacia las 13,20 del mismo día 24 aparecieron sobre la coronación unos penachos de polvo que se elevaban hasta casi cinco metros, restos de los cuales se aprecian, aunque con dificultad, en la fotografía 1 (señalados con A). Estos se originaron por la caída dentro de la grieta del detritus que, procedente de antiguos laboreos, cubre parte de la coronación con espesores de hasta un metro, y eran indicativos de que la grieta se estaba ensanchando. El colapso era inminente y efectivamente comenzó unos minutos después, en la hora registrada por el Observatorio Sismológico, con desprendimientos laterales de material fragmentado similares al señalado con B en la fotografía 1; la mayoría de este material se acumuló al pie formando un talud de 35 grados de inclinación, aunque fragmentos no superiores a $50 \mathrm{~cm}$, y en cantidad relativamente pequeña (fotografía 2), se dispersaron radialmente hasta una distancia máxima de 80 metros. Cuatro segundos después de [34] iniciado este proceso ocurrió la rotura general del prisma rocoso recogida en la fotografía 1.

ASPECTOS DINÁMICOS.- El sistema de diaclasas forma un ángulo aproximado de $15^{\circ}$ respecto al frente, mientras que el de fallas incide a unos $30^{\circ}$ (fig. 2), por lo que cabría esperar una apertura del primero por vuelco dada su mayor ortogonalidad con el vacío. Sin embargo ya se ha indicado que las grietas preexistentes se abrieron siguiendo el sistema de fallas, lo que puede explicarse admitiendo la existencia de tensiones internas, de origen tectónico, orientadas según un eje de comprensión N-S o de tracción E-W. Ambos ejes son posibles ya que el primero coincide con el de comprensión de las Cadenas Béticas durante el Cuaternario, confirmado en el Campo de Alicante por observaciones aún inéditas del autor, y el segundo con el de tracción que originó la falla en la que se encajó el antiguo Barranco de Bonivern separando el Benacantil de la Sierra de San Julián. Cualquiera de los dos, o la conjunción de ambos, pueden explicar la existencia de una anisotropía tensional, disipada en las inmediaciones del frente al ser posible allí los desplazamientos, aunque una comprensión N-S se traduciría durante la distensión en una apertura selectiva del sistema de diaclasas $\mathrm{N}-60^{\circ}-\mathrm{W}$, por lo que es más admisible la hipótesis de tracciones E-W. Estas producen la apertura de los labios de una de las fallas dando lugar a un desplazamiento hacia el Oeste del prisma y obligando a las diaclasas a actuar como fallas horizontales dextrógiras, con lo que una parte de los enlaces y rugosidades en el plano de la diaclasa activa son eliminados, quedando la masa deslizante en algún punto de la rama ascendente de la curva esquematizada en la figura 3. Este tipo de curvas fue interpretado inicialmente por Skempton (1965) a partir de ensayos sobre suelos e inmediatamente utilizado para explicar el proceso de «rotura progresiva» en macizos rocosos por Müller (1967). Al 
iniciarse el desplazamiento a lo largo de un plano de discontinuidad en la roca, se van produciendo encajes entre las rugosidades superficiales, lo que hace aumentar la tensión cortante necesaria para continuar el movimiento dando el tramo ascendente de la curva; para un cierto desplazamiento la tensión alcanza un máximo, la «resistencia pico», a partir de la cual las rugosidades quedan laminadas y la tensión [35] cae hasta alcanzar la resistencia «residual», equivalente a la que ofrecen dos caras lisas de la roca en cuestión deslizando una respecto a la otra.

En el caso aquí estudiado, el prisma debió quedar estabilizado en la rama ascendente pese a esos desplazamientos de hasta $30 \mathrm{~cm}$., pero la acción del agua infiltrada entre los días 9 y 11 modificó los parámetros resistentes facilitando la rotura de unos «puentes» y sobrecargando los restantes hasta que, progresivamente, se sobrepasó el pico de resistencia.

Es difícil evaluar esa acción del agua ya que no se realizó ningún ensayo «in situ» habida cuenta del enorme dispendio económico que estos implican y que sólo los hacen rentables en el caso de riesgo muy elevado (túneles, presas, etc.) pero pueden estimarse los siguientes factores:

a).- Carga hidrostática.- En el caso de inundación de las grietas, con sus treinta metros de penetración vertical hasta la discordancia, se alcanzarían en la base presiones máximas de 3,0 $\mathrm{kg} / \mathrm{cm}^{2}$ cuya componente horizontal actuaría en el sentido de despegar el prisma del macizo. Ahora bien, admitiendo una anchura media de diez centímetros para las grietas, habrían caído directamente sobre éstas tan sólo 6 litros por metro lineal, necesitándose en estas circunstancias una cuenca de recepción de $50 \mathrm{~m}^{2}$ por cada uno de esos metros lineales de grieta para producir el llenado, pero teniendo en cuenta que los $60 \mathrm{~mm}$. cayeron durante tres días, con lo que tendría lugar un drenaje parcial, no parece muy probable que se llegase a ese estado de presiones máximas.

b).- Fuerzas de filtración.- Al igual que las cargas hidrostáticas sólo pudieron actuar durante los primeros días después de las lluvias, pero en el mejor de los casos, y teniendo en cuenta lo dicho anteriormente, los gradientes nunca alcanzarían valores suficientes como para generar fuerzas de filtración superiores a algunas decenas de gramos por centímetro cuadrado.

c).- Hidroexpansividad.- Birot (1981, p. 84) proporciona valores del coeficiente de hidroexpansividad para calizas [36] del orden de 5,0. 10 $0^{-5}$. Si esta expansión está impedida se originarán compresiones que con el módulo de elasticidad de la calcarenita (Cuadro I) alcanzarán $\operatorname{los} 8 \mathrm{~kg} / \mathrm{cm}^{2}$, carga ésta muy lejana a la necesaria para producir la rotura, pero sí elevada en cuanto a aumentar las tensiones efectivas en el plano de rotura y colaborar con la estabilidad ya que la expansión se produce por igual en ambos lados del plano de discontinuidad.

d).- Disolución.- La elevada porosidad de la calcarenita de la Sierra de San Julián la hace muy susceptible a fenómenos de corrosión interna diferencial como puede apreciarse en numerosas fachadas de edificios construidos en Alicante con dicha roca. No hay datos para evaluar esa disolución en el caso aquí estudiado, pero presumiblemente el agua habrá circulado a través de las grietas durante años degradando la roca y reduciendo la resistencia de los puntos de apoyo.

e).- Termoclastia.- Es un proceso tan citado en la bibliografía como poco analizado respecto a su importancia morfogenética real fuera del laboratorio, motivo por el cual se tratará aquí con cierto detalle.

En la figura 4a se han representado las temperaturas máximas (A) y mínimas (B) entre los días 9 a 24 de noviembre, tomadas de los registros termográficos de la Universidad de Alicante, y en la $4 \mathrm{~b}$ los gradientes de elevación diaria expresados como el cociente entre la diferencia de temperaturas extremas y el tiempo, en horas, empleado para pasar de la mínima a la máxima. Puede apreciarse como no existen grandes amplitudes en la variación térmica capaces de justificar fenómenos termoclásticos, pero sí es muy significativo el brusco aumento del gradiente ocurrido el día 24 de forma que se pasa de la mínima a la máxima con una variación 
de $2,4^{\circ} \mathrm{C} /$ hora. Para evaluar la influencia de ese factor se han calculado, a partir de las bandas del termógrafo, los perfiles términos dentro de la roca a las 7,00, a las 10,00 y a las 13,20 (TMG) obteniéndose las curvas de la figura 5. La metodología está basada en la ecuación propuesta por Cermak (1971) a partir de la indicada por Birch (1948) que se expresa:

$\mathrm{T}_{\mathrm{z}}=\mathrm{T}_{\mathrm{o}}\left(\operatorname{erf} \mathrm{n}_{\mathrm{i}}+1-\operatorname{erf} \mathrm{n}_{\mathrm{i}}\right)[37]$

en la que:

$\mathrm{T}_{\mathrm{z}}=$ Temperatura a la profundidad $\mathrm{z}(\mathrm{cm})$

$\mathrm{T}_{\mathrm{o}}=$ Temperatura en superficie, $\left({ }^{\circ} \mathrm{C}\right)$.

$\mathrm{n}=\mathrm{z}(4 \mathrm{kt})^{-1 / 2}$

$\mathrm{t}=$ Tiempo de aplicación de la temperatura $\mathrm{T}_{\mathrm{o}}(\mathrm{seg})$,

$\mathrm{k}=$ Coeficiente de difusión térmica $\left(0,011 \mathrm{~cm}^{2} / \mathrm{seg}\right)$.

Se observa como la curva A (7,00 TMG) registra la influencia del descenso térmico nocturno aunque iniciando una recuperación en los dos primeros centímetros. La B (10,00 TMG) ya muestra una recuperación superficial pero acusando el mínimo nocturno hacia los $15 \mathrm{~cm}$. de forma que solamente los diez primeros centímetros indican aquel pequeño aumento. Por último la $\mathrm{C}$, cinco minutos antes del desprendimiento, presenta una distribución exponencial centrales del día, aunque ya se había iniciado un ligero descenso en superficie desde la máxima alcanzada a las 12. Con las diferencias de temperatura entre las curvas A y C, el módulo de elasticidad de la calcarenita (Cuadro I) y un coeficiente de dilatación cúbica de 2.7. 10 $0^{-5}$ (Perry, 1966) se obtiene la distribución de tensiones térmicas representada en la figura 6 (curva A) siempre y cuando los desplazamientos de dilatación se encuentren impedidos, hecho este que se producirá en los «puentes» de enlace entre las caras de la diaclasa según se esquematiza en la figura 7. De acuerdo con este modelo la cámara de aire entre ambas caras interrumpe la onda térmica del prisma mientras que la propagación de ésta desde el frente del macizo lateralmente hacia la cara interior de la diaclasa progresa más lentamente a causa del elevado valor del gradiante de variación, creando una «zona de sombra» donde se presentarán las tensiones térmicas diferenciales; lo mismo ocurrirá si la diaclasa se encuentra rellena de material suelto, pues para éste el autor ha determinado valores del coeficiente de difusión térmica comprendidos entre 2,3.10-4 y 3,0.10 $\mathrm{cm}^{2} / \mathrm{seg}$. por lo que frenará la propagación aunque en menor medida. Puede apreciarse como hasta la profundidad de $15 \mathrm{~cm}$ la tensión térmica supera a la resistencia a tracción de la calcarenita, evaluada en $13 \mathrm{~kg} / \mathrm{cm}^{2}$ (Cuadro I). Otro factor que parece no haber sido tenido en cuenta hasta ahora por los geomorfólogos es la aparición de presiones intersticiales de origen térmico. Si en una roca saturada se propaga una onda térmica con una velocidad superior a [38]

\section{CARACTERÍSTICAS MECÁNICAS DE LA CALCARENITA DE SAN JULIÁN}

Peso específico

$2,28 \mathrm{~g} / \mathrm{cm}^{3}$

Absorción de agua (a presión atmosférica)

$4,89 \%$

Resistencia a compresión simple

$167 \mathrm{~kg} / \mathrm{cm}^{2}$

Módulo de elasticidad

$160.000 \mathrm{~kg} / \mathrm{cm}^{2}$

(1).- Muestras de los niveles más cementados elevan este valor hasta $28 \mathrm{~kg} / \mathrm{cm}^{2}$

la que es capaz de producir el gradiante hidráulico generado por la mayor expansión del agua respecto a la caliza (20 a 1 aproximadamente) aparecen fuerzas de expansión intersticiales 
similares a las que se producen al ensayar probetas de roca saturada con cargas rápidas, las cuales rompen con descensos de resistencia de hasta un $50 \%$ respecto a las de roca seca. El tratamiento teórico es complejo pero puede calcularse que para el perfil térmico dado por la curva $\mathrm{C}$ de la fig. 5 se obtendrá la distribución de tensiones máximas representadas por la curva B en la fig. 6. Es un fenómeno que sólo puede ocurrir en aquellas zonas de la roca con coeficientes de permeabilidad muy pequeños pero que pudo contribuir a la rotura en puntos localizados; tanto en apoyo de esto como en el de la hipótesis de tensiones térmicas diferenciales en roca seca, expuesto anteriormente, puede señalarse el hecho de que casi todas las caídas de piedras y pequeños desprendimientos, se produjeron principalmente a partir de media mañana y hasta primera hora de la tarde, cuando el sol comenzaba a incidir directamente en el frente de cantera. A este respecto, Goudie (1981), citando trabajos de otros autores indica que el máximo de desprendimientos en las Montañas Rocosas Canadienses se produce entre las siete y las nueve, y entre las doce y las dieciséis. No se indica la orientación de las laderas, pero es sintomático que coincidan con el inicio y con el máximo de la insolación.

A la vista de los procesos expuestos, parece que los más significativos son los de disolución y los termoclásticos actuando los primeros a largo plazo y colaborando los segundos en los momentos finales. Una vez sobrepasado el pico de resistencia en el plano de rotura se iniciaron los desprendimientos laterales ya mencionados que dejaron en libertad al prisma principal, ocurriendo entonces como [39] si todo el peso de dicho prisma hubiese gravitado sobre su pie, en la discordancia, iniciándose en ella un plano de corte inclinado $80^{\circ}$ hacia el frente y con una superficie de $15 \mathrm{~m}^{2}$ tal y como pusieron de manifiesto medidas posteriores. Teniendo en cuenta que el peso del material desplomado fue de $2.800 \mathrm{Tm}$, las tensiones normal $\left({ }^{\sigma} \mathrm{n}\right) \mathrm{y}$ tangencial $\left({ }^{\sigma} \mathrm{t}\right)$ en el plano de rotura fueron:

${ }^{\sigma} \mathrm{n}=3,2 \mathrm{~kg} / \mathrm{cm}^{2}$

${ }^{\sigma} \mathrm{t}=18,4 \mathrm{~kg} / \mathrm{cm}^{2}$

Aplicando la fórmula Hoek (Hoek, 1970, P. 119) para determinar la resistencia al esfuerzo cortante (s) en función de la resistencia a compresión simple y de la tensión normal se obtiene:

${ }^{\sigma} \mathrm{s}=21,1 \mathrm{~kg} / \mathrm{cm}^{2}$

valor este comparable en magnitud al de ${ }^{\sigma} \mathrm{t}$ determinado anteriormente, lo que apoya la hipótesis de que en el último momento todo el prisma quedó aislado de la pared, descansando su peso en la discordancia que rompió ante esa sobrecarga.

En resumen el proceso debió seguir estas pautas:

a).- Apertura del sistema de fallas por efecto de tensiones residuales obligando al sistema de diaclasas a actuar como pequeñas fallas horizontales dextrógiras. (fig. 2). Así quedarían anulados muchos de los enlaces internos que mantenían la estabilidad.

b).- Infiltración de agua que, con el tiempo, fue debilitando los enlaces todavía existentes hasta que las lluvias de los días 9 a 11 culminaron el proceso de degradación y el plano de rotura sobrepasó la resistencia pico.

c).- A partir de ahí se inicia la rotura progresiva ensanchándose las grietas y produciéndose pequeños desprendimientos, especialmente en la arista NW del prisma, donde se concentrarían las tensiones de origen térmico.

d).- Anulados los enlaces entre prisma y macizo a través de las grietas ensanchadas, aquél quedó totalmente apoyado sobre su pie, en la discordancia, dando lugar a una sobrecarga que formó un plano de rotura independiente de [40] las grietas y discontinuidades anteriores, 
originando la caída prácticamente vertical del prisma.

CONSECUENCIAS GEOMORFOLÓGICAS.- A la escala del macizo el desprendimiento tuvo incidencia mínima, limitada a la aparición de una superficie netamente más regular que la del resto del frente, localizada según el plano de diaclasa, en la cual se aprecian zonas con estrías semejantes a las de falla pero de corta longitud, apenas algunos decímetros como máximo. Más importancia tiene un hecho menos aparente consistente en que tras el desprendimiento se aceleró, al menos momentáneamente, la apertura de una grieta, siguiendo de nuevo el sistema de fallas, algo más al NW, que individualizó un nuevo prisma con amenaza de próxima ruptura. Es evidente que el concepto de «rotura progresiva» acuñado por Müller no debe limitarse al plano de deslizamiento, sino que puede hacerse extensivo en el tiempo a zonas próximas que se verán afectadas por la nueva distribución de tensiones, de forma que los desprendimientos se sucederán a intervalos imposibles de estimar, tendiendo a regularizar la ladera hasta alcanzar un cierto estado de equilibrio.

Mayor importancia tiene la acumulación de fragmentos al pie del talud (fotografía 2 y 3 ). Estos formaron un semicono de 35 metros de diámetro por 12 de altura, lo que da un volumen de $2.000 \mathrm{~m}^{3}$ y un peso de $2.800 \mathrm{Tm}$, equivalentes a $1.200 \mathrm{~m}^{3}$ de roca «in situ». Lo que podemos considerar como núcleo de este semicono está formado por el material desprendido en los primeros segundos y consiste en clastos poliédricos de granulometría continua desde un máximo de 50-60 cm. hasta el tamaño de la arena fina, sin estratificación aparente y con un ángulo de talud comprendido entre $35^{\circ}$ y $38^{\circ}$, mensurable en el extremo SE donde no fue «aplastado» por la masa principal. Una pequeña parte de estos fragmentos adquirieron un cierto momento angular durante la caída, lo que hizo que se extendieran en abanico por rodamiento hasta una distancia máxima de ochenta metros como ya se ha indicado.

Sobre este núcleo cayó la masa principal del prisma que se fragmentó en bloques de volumen individual medio entre uno y dos metros cúbicos, los cuales quedaron materialmente clavados en el núcleo y encajados entre sí, no produciéndose [41] dispersión a causa de la horizontalidad de la base y al hecho de que el desplome fue prácticamente vertical, sin componentes de vuelco, tal y como se aprecia en la fotografía 1.

CONCLUSIONES FINALES.- Lo hasta aquí expuesto sirve para tomar en consideración algunos factores que, aun no siendo nuevos, rara vez son apreciados al estudiar los fenómenos asociados a las laderas.

En primer lugar es de destacar la influencia de las tensiones internas en cuanto a la orientación de los desplazamientos relativos que ocurren en las inmediaciones de los cantiles rocosos, tema sobre el Gaudie (1981) escribía: «The mechanics of very-high-magnitude rocks failures are poorly understood and opinions differs about the relative importance of structural elements and residual stresses in the rock due to tectonic activity». La medición de estas tensiones es muy sencilla pero a la vez muy cara, motivo éste por el que normalmente escapa a las posibilidades de la investigación pura, aunque en muchos de los puntos donde han podido medirse se han obtenido valores sorprendentemente elevados (Lo y Lee, 1975); ahora bien, pueden estudiarse cualitativamente analizando los sistemas de diaclasas en las zonas inmediatas a los cantiles, proporcionando valiosas indicaciones sobre el estado de tensiones tectónicas a que se ve sometido el macizo, capaces de alterar el modelo dinámico de rotura estimado como más probable tal y como se ha comprobado en la Sierra de San Julián.

Por otra parte la acción del agua se presenta como decisiva en una zona libre de heladas como es Alicante y además exenta de vegetación que pueda influir con sus raíces. La coincidencia de las lluvias de los días 9 a 11 con el comienzo de los desprendimientos así como el incremento del gradiente el día de la rotura difícilmente pueden considerarse casuales, por lo 
que fenómenos tales como corrosión interna (incluyendo una posible acción haloclástica no comprobada), termoclastia y presiones intersticiales de origen térmico deberían ser investigadas con mayor profundidad para tratar de explicar la clastogénesis en áreas similares del litoral mediterráneo.

En cuanto a las consecuencias geomorfológicas directas [42] pueden considerarse como discretas pero muy aleccionadoras si se tiene en cuenta la fuerte fragmentación ocurrida en los primeros instantes del desprendimiento y que dio origen a materiales con «facies de canchal» seguidos por bloques de tamaño ínfimo si se los compara con el volumen movilizado (relación de 1 a 1.000 aproximadamente), lo que obliga a investigar desde este punto de vista algunos de los canchales de cotas bajas, ya que la progresión lateral de la inestabilidad observada en la Sierra de San Julián bien puede haberse producido en otros cantiles dando una coalescencia de taludes al pie, especialmente enmascarados si una ladera inclinada ha permitido la dispersión por rodamiento hacia abajo de los bloques más gruesos.

\section{BIBLIOGRAFÍA}

Birch, F.- 1948.- «The effects of Pleistocene climatic variations upon geothermal gradients». Amer. Jour. of Science, v. 246, pp. 729-760.

Birot, P.- 1981.- «Les processus d'érosion a la surface des continents». Ed. Masson, París.

Cermak; V.- 1971.- «Underground temperature and inferred climatic tempereture of the past millenium». Paleog. Paleoclim. Paleoec., v. 10, pp. 1-19.

Gaudie, A.- 1981.- «Geomorphological Techniques». Brithis Geomorphological Research Group, Londres.

Hoek, E.- 1970 «Rotura frágil de las rocas», en Mecánica de Rocas en la ingeniería práctica, Ed. Blume, Madrid, pp. 103-124.

Lo, K. Y. and Lee, C. F.- 1975.- «Stress distribution in rock slopes under high in situ stresses», en Mass wasting, University of West Anglia, Norwich, pp. 35-55.

Montenat, C.- 1975.- «Le Neogene des Cordilleres Betiques. Essai de synthese stratigraphique et paleogeographique», B.E.I.C.I.P., París.

Müller, L.- 1967.- «La rotura progresiva en roca diaclasada», Trad. del Bol. Inf. del Lab. del Transporte y Mecánica de Suelos, C.E.O.P., nº 55, pp. 31-42.

Perry, J. H.- 1966.- «Manual del Ingeniero Químico», UTEHA., México.

Ramos Hidalgo, A.- 1983.- «Los mecanismos y los procesos de inundación en la Ciudad de Alicante», en Lluvias torrenciales e inundaciones en Alicante, Inst. Univ. de Geografía, Univ. de Alicante, pp. 73-98.

Ramos Hidalgo, A.- 1984.- «Evolución urbana de Alicante». Inst. de Est. «Juan Gil-Albert», Excma. Diputación Provincial, Alicante.

Skempton, A. W.- 1965.- «Estabilidad de taludes de arcilla a largo plazo», Trad. del Bol. Inf. del Lab. del Transporte y Mecánica de Suelos, C.E.E.O.P., Madrid, no 38, pp. 67-101. [43] 


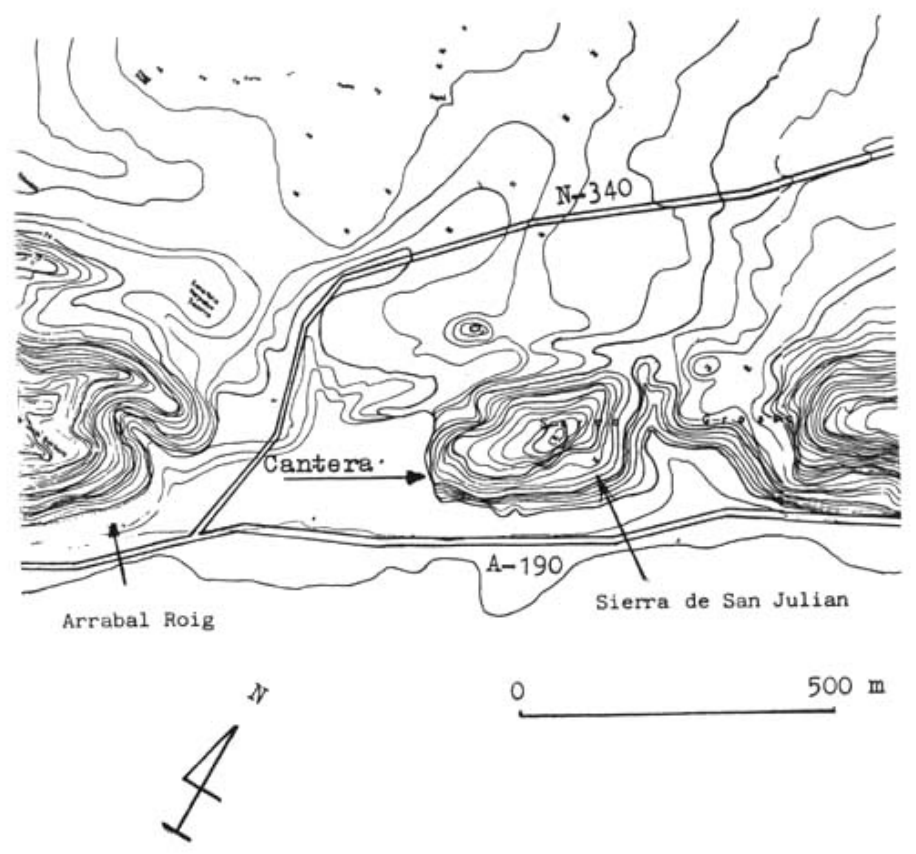

Gráfico 1.- Plano de situación

[44]

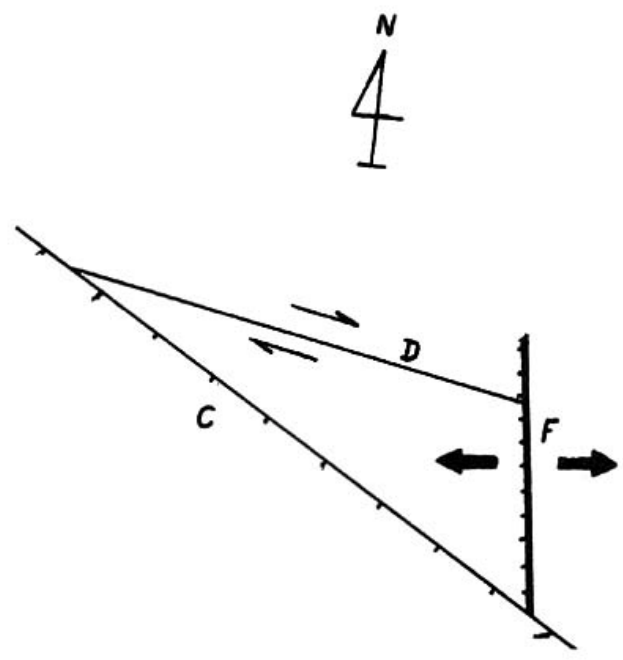

Gráfico 2.- Esquema dinámico en el frente de cantera. C.- Frente; D.- Diaclasa movilizada como falla dextrógira; F.- Falla en tracción 


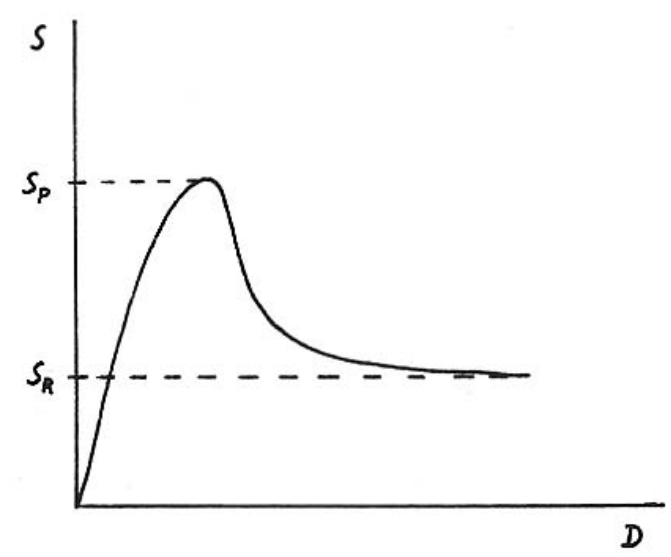

Gráfico 3.- La tensión de corte (S) como función del desplazamiento (D), exponiendo los conceptos de resistencia pico $\left(\mathrm{S}_{\mathrm{p}}\right)$ y resistencia residual $\left(\mathrm{S}_{\mathrm{R}}\right)$

[45]

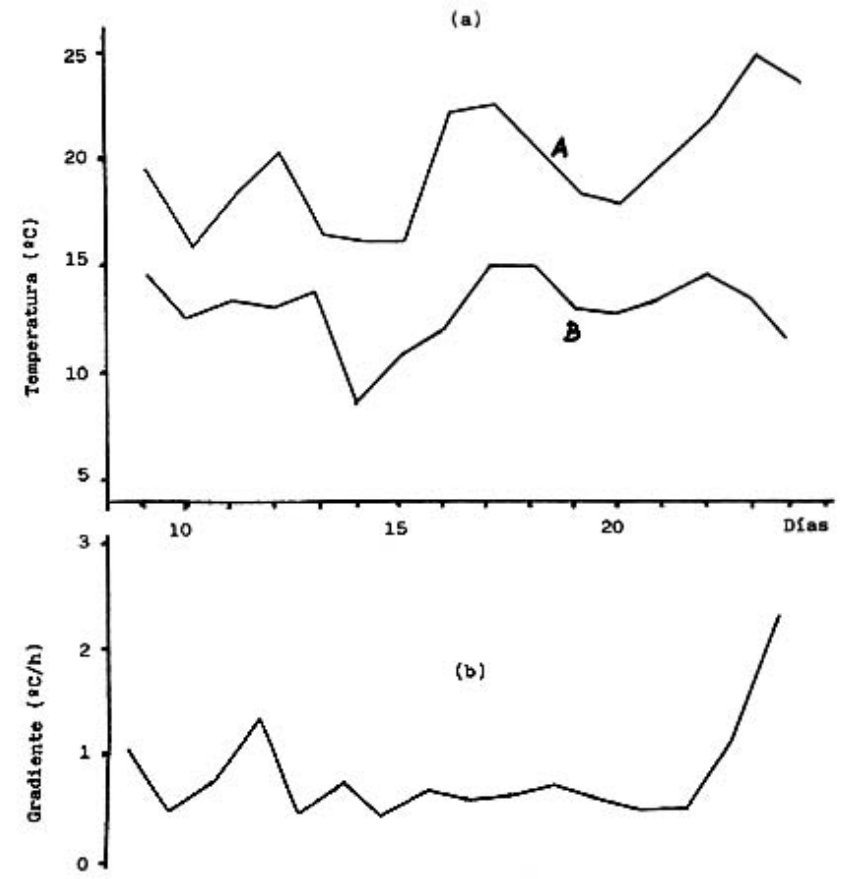

Gráfico 4.-(a). Temperaturas máximas y mínimas (A y B respectivamente) entre los días 9 y 24 de noviembre (b). Velocidad de ascenso desde la temperatura mínima a la máxima. Es evidente el disparo hacia arriba iniciado [46] el día 23 y acelerado el 24 


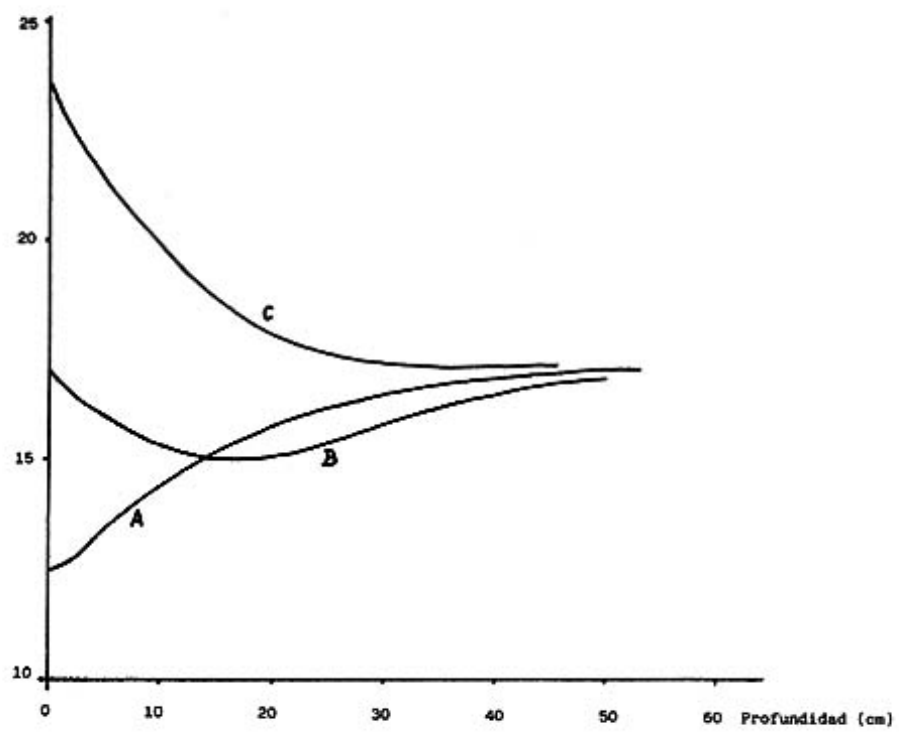

Gráfico 5.- Perfiles térmicos en la roca, a partir del frente, a las 7,00 (curva A); 10,00 (curva B) y 13,20 (curva [47] C) $\mathrm{TMG}$

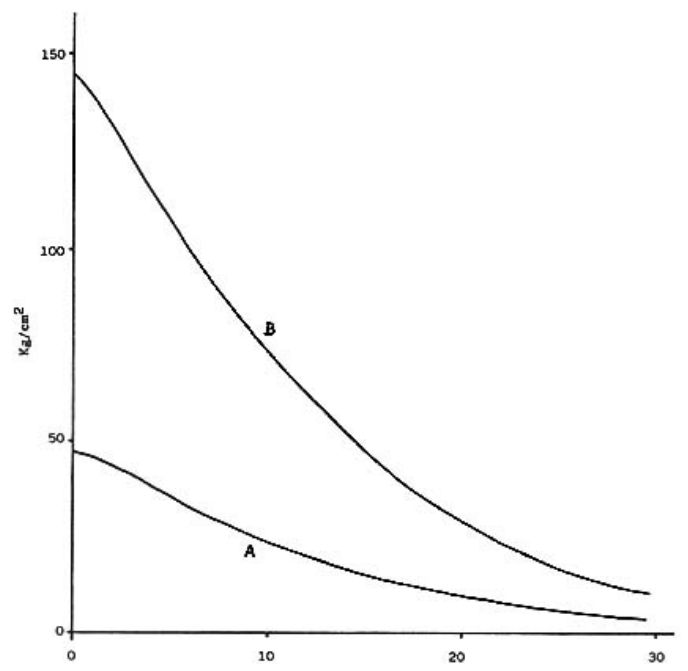

Gráfico 6.- Tensiones máximas de dilatación en la roca (curva A) e intersticiales (curva B) 


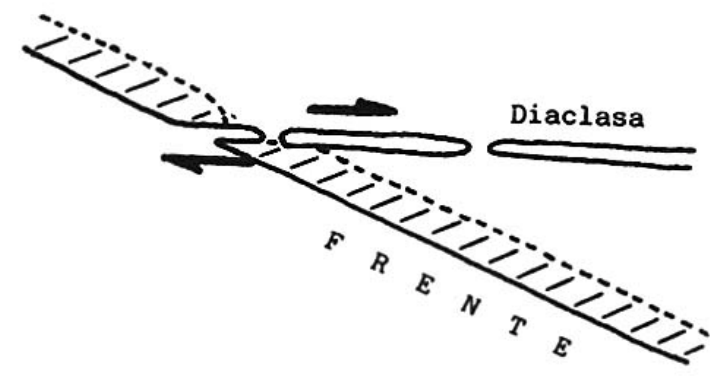

Gráfico 7.- En rallado oblicuo las zonas sometidas a dilatación. Se aprecia el «efecto de sombra» originado por la diaclasa y el par de corte por él inducido (flechas)

[48]

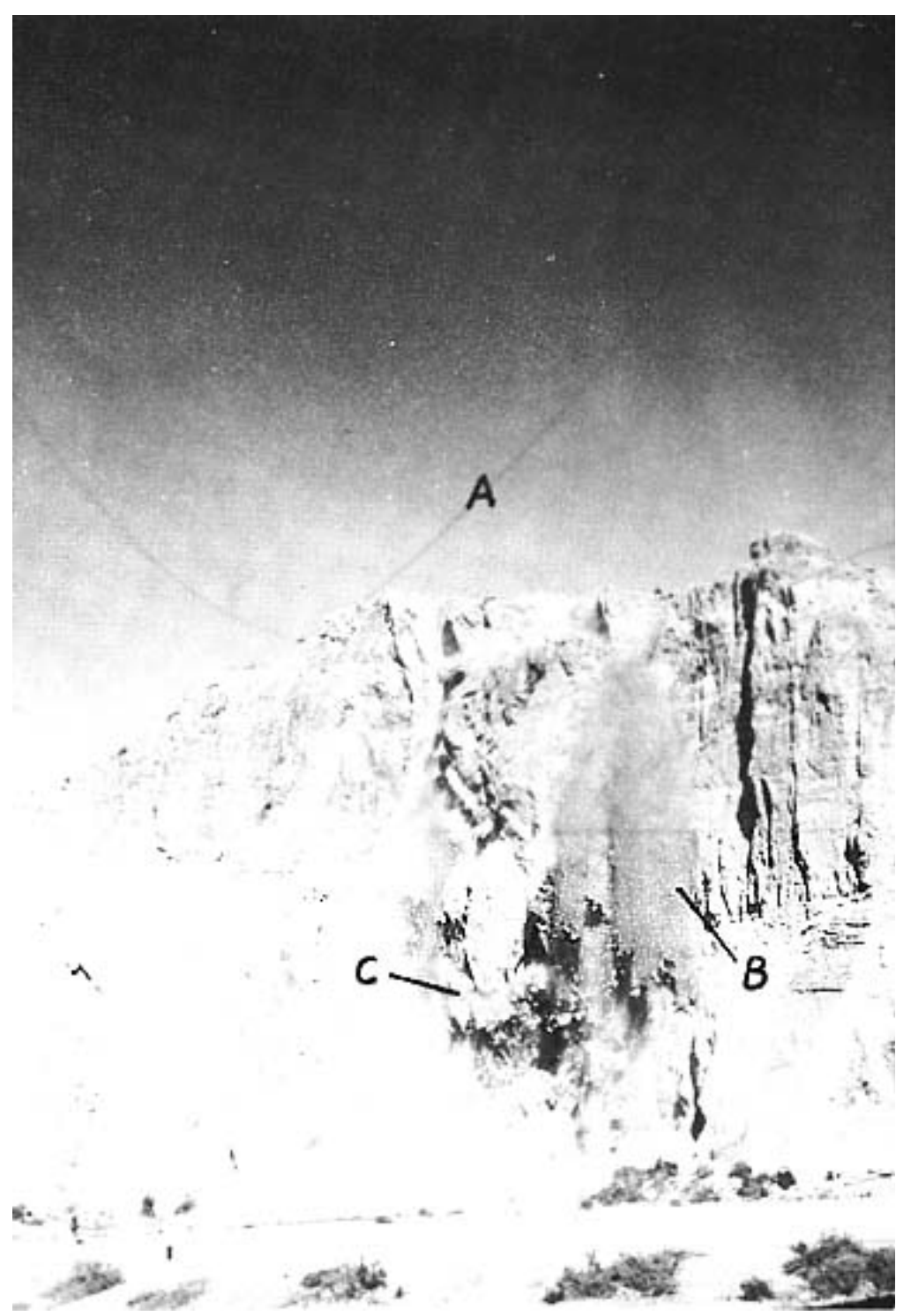

Figura 1.- Instantánea del desprendimiento. Obsérvese como la rotura se produce en el pie (señalado con C) [49] 


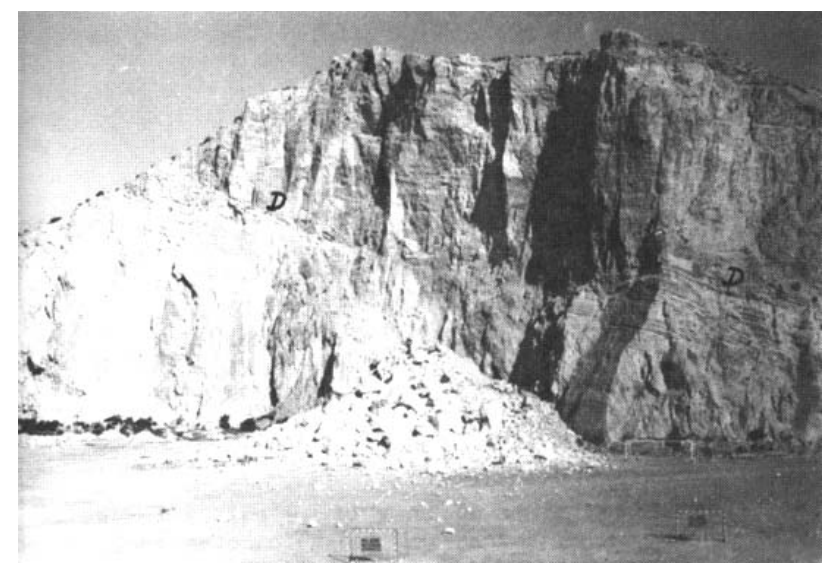

Figura 2.- Cono de derrubios formado por el desprendimiento. Se aprecian claramente la discordancia (D) y unos carteles anunciando el peligro colocados después del suceso

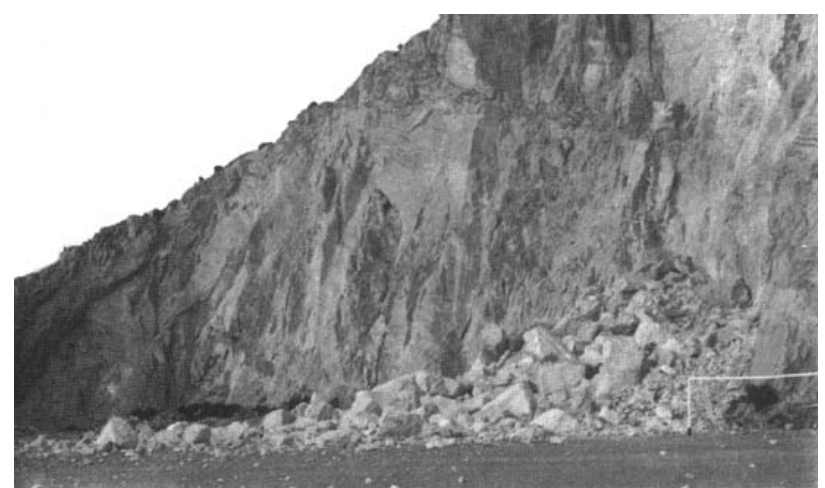

Figura 3.- Vista lateral del cono de derrubios. Se aprecia el plano de rotura al nivel de la discordancia 